\title{
A Novel Controller Dynamic Linearization-Based Model Free Adaptive Predictive Control for A Class Of Discrete Nonlinear Systems
}

\author{
Danna Wang \\ School of Electronic and Information Engineering, Beijing Jiaotong University, Beijing, China \\ 15120278@bjtu.edu.cn
}

\begin{abstract}
Keywords: Model Free Adaptive Control; Data Driven Control; Model Free Adaptive Predictive Control; Controller Dynamic Linearization.
\end{abstract}

\begin{abstract}
In this work, a novel controller dynamic linearization based model free adaptive predictive control algorithm (CFDLc-MFAPC) for a class of discrete nonlinear system is proposed. This method introduces dynamic linearization parameters of ideal controller based on dynamic linearization technology, and combines predictive control idea to realize predictive function in controller. Compared with the model-based control method, the advantage of this method is mainly that it is a pure data driven control (DDC) method, and does not need any model information, but only needs the I/O data of the system. Moreover, the introduction of predictive control ideas not only improves the robustness of the system, but also realizes the model free adaptive prediction effect for the expected mutation. By this method, the robustness of the algorithm can be improved. The validity of the proposed method is verified by numerical simulations.
\end{abstract}

\section{Introduction}

With the development of information science and technology, more complex equipment was born, production equipment to the large-scale, complex development, based on the traditional theory and method of model control is widely used in various industries, but the control effect and its accuracy in many cases cannot satisfy people's control requirements, especially in the control of some difficult modeling system, it is more weak.

Due to the development of modern electronic technology, most of the industrial production processes produce huge amounts of production data every day, and the real operating information of the system is contained in these data. How to control the input and output data of the controlled object directly and effectively. How to reduce the dependence of the control system on the mathematical model of the controlled object and how to improve the control system is discussed, the robust performance of these systems is an urgent problem to be solved in the industrial field.

In this case, data driven control method becomes a feasible method, which can effectively avoid using the traditional model only depends on the input and output (I/O) measurement data of the controlled plant. Until now, many kinds of DDC approaches have emerged [1-4]. there are a variety of data-driven control methods, e.g. such as model-free adaptive control (MFAC) [5], lazy learning control (LL) [6], virtual reference feedback tuning (VRFT) [7], iterative feedback tuning (IFT) [8], correlation-based controller tuning (CbT) [9], etc. Among above mentioned data driven control methods, the model free adaptive predictive control (MFAPC) [10] approach, which has been widely used in recent years, e.g., the predictive function is implemented in the controller using a lazy-learning (LL)-based model free adaptive predictive algorithm[11], control of oxygen enriched air Concentration[12] etc.

The existing researches about this topic mainly focus on the model free adaptive control systems. However, for the model free adaptive predictive control system, the controller dynamic linearization is still an open problem. In this paper, we consider the controller dynamic linearization of MFAPC and give the ideal controller structure and time-varying parameters of the iedal controller.

In this work, a novel model free adaptive predictive control method based on controller dynamics linearization. The main feature of the proposed method is that, two equivalent dynamic linearization 
data models, one is on the generic ideal controller to a general discrete-time nonlinear system and the other is on the controlled plant, are used to design and analysis the compact form dynamic linearization based model free adaptive predictive controller (CFDLc-MFAPC) scheme, and neither the controlled plant model nor the structure information are involved in the controller design and analysis. The time varying pseudo-partial derivative (PPD) is tuned by an optimization method on a given controller designing criterion function using on-line measured $\mathrm{I} / \mathrm{O}$ data of the controlled plant in close loop. Moreover, the introduction of predictive control ideas, such that the controller not only shows good robustness but also can realize the effect of model-free adaptive prediction for the sudden change of the desired signal. This method not only avoids the modeling of the controlled object, but also has strong robustness. Numerical simulations verify the effectiveness of the proposed method.

The rest of the paper is organized as follow: Section II introduces the main result of the new type of MFAPC method, including description of CFDLc data model, both on ideal controller and controlled plant, controller designs. Then, the PPD in CFDLp and the parameters in the CFDLc are estimated in section III. The numerical examples are given in the section IV and Section IV concludes the work.

\section{CFDLc-MFAPC Controller Design}

In this section, the dynamic linearization technology and the controllers dynamic linearization technology are introduced in the part $\mathrm{A}$ and the part B, respectively. On this basis, the part $\mathrm{C}$ introduces the design of model free adaptive predictive control based on the dynamic linearization of the controller.

\subsection{Dynamic Linearization Technique}

Consider a class of SISO nonlinear discrete-time systems described by

$$
y(k+1)=f\left(y(k), \cdots, y\left(k-n_{y}\right), u(k), \cdots, u\left(k-n_{u}\right)\right)
$$

Where $n_{y}, n_{u}$ are the unknown positive constants of the output $y(k)$ and the input $u(k)$ respectively, $f(\cdots)$ is an unknown nonlinear function.

Ala. The partial derivative of $f(\cdots)$ with respect to the $\left(n_{y}+2\right)^{\text {th }}$ variable is continuous, for all $k$ with finite exceptions.

A2a. System (1) satisfies generalized Lipschitz condition, for all $k$ with finite exceptions, that is, $\left|y\left(k_{1}+1\right)-y\left(k_{2}+1\right)\right| \leq b\left|u\left(k_{1}\right)-u\left(k_{2}\right)\right|$,

For $u\left(k_{1}\right) \neq u\left(k_{2}\right)$ and any $k_{1} \neq k_{2}, k_{1}, k_{2} \geq 0$ where

$$
y\left(k_{i}+1\right)=f\left(y\left(k_{i}\right), \cdots, y\left(k_{i}-n_{y}\right), u\left(k_{i}\right), \cdots, u\left(k_{i}-n_{u}\right)\right), i=1,2 \text {; and } b \text { is a positive constant. }
$$

Lemma 1[10] Consider nonlinear system (1) satisfying A1 and A2. If $|\Delta u(k)| \neq 0$, then there exists a time-varying parameter $\phi(k) \in R$, called pseudo partial derivative (PPD), such that system (1) can be transformed into the following CFDL data model,

$$
\Delta y(k+1)=\phi(k) \Delta u(k)
$$

With $|\phi(k)| \leq \bar{b}$ for any $k$ under the case of linearization length constant (LLC) $L=1$.

Based on above equivalent incremental data model, the following one-step-ahead prediction equation can be got straightforwardly

$$
y(k+1)=y(k)+\phi(k) \Delta u(k)
$$

Compared with other linearization methods for nonlinear function, this dynamic linearization method possesses the following features. First, it does not require the mathematics model, order and time delay of the controlled plant. Second, it is an equivalent dynamic linearization data model rather than a static approximation model and no high-order term is dropped. Third, the dynamic linearization model, having time-varying incremental from with very simple structure and very few parameters, is a data model only for the purpose of controller design rather than first principles model. The introduction of linearization length constant can avoid the high-order controller design. In 
CFDLp data model, all of the nonlinear properties and the parameter estimation error are fused into the scalar PPD, thus the dynamic behavior of the PPD may be very complicated, and, as a result, it would be difficult to design an algorithm to capture this complex dynamic behavior. Finally, the dynamic behavior of pseudo-partial derivative is not sensitive to the time-varying parameter, structure, or delay of a controlled plant, but these factors explicitly formulated in the first principles model or the identified model, in which they are hard to handle.

Through dynamic linearization technology, the nonlinear system (1) is equivalent to the data model (2). In the sequel, we will forcos on controller dynamically linearization.

\subsection{Controller Dynamic Linearization}

For nonlinear system (1), assume that there exists an ideal nonlinear controller of the following from (4), which can stabilize the plant (1) and drive the plant output to track the desired signal asymptotically.

$$
u(k)=C\left(u(k-1), \cdots, u\left(k-n_{c}\right), e(k+1), \cdots, e\left(k-n_{e}\right)\right),
$$

Where $C(\cdot)$ is a smooth unknown nonlinear function, $u(k)$ is the control input, and $e(k)=y_{d}(k)-y(k)$ is the output tracking error at time instant $k ; y_{d}(k), y(k)$ are the desired and actual output of the controller plant, respectively; $n_{c}, n_{e}$ are the two unknown orders of the controller[13]

In this section, a novel controller design method, that is, controller compact form dynamic linearization based MFAPC method (CFDLc-MFAPC), is proposed for controlled plant (1).

Different from the prototype MFAC, the proposed method is based on the equivalent dynamic linearization method both on the ideal controller and on the controlled plant, which can be realized merely using the I/O data of the controller and of the controlled plant.

Before presenting the details, some assumptions are made as follows.

Alb. The controller (4) is a smooth nonlinear function, and the partial derivatives of $C(\cdot)$ with respect to error input signals $e(\bullet)$ are continuous.

A2b. The system (4) is generalized Lipschitz, that is, for any $|\Delta u(k)| \leq \underline{b}_{\mid}|\Delta e(k+1)|$ is satisfied for any time instant $k$ and $|\Delta e(k+1)| \neq 0$. Where $\underline{b}$ is a positive constant, and $\Delta e(k+1)=e(k+1)-e(k)$, $\Delta u=u(k)-u(k-1)$.

Remark 1: Similar to other data-driven control methods, it is assumed that A1 is the general condition of the ideal controller.

Suppose that A2 is a bounded constraint on the influence of the output tracking error increment on the control input increment, which indicates that the controller is stable.

The following theorem indicates that the general discrete time non-linear system satisfies assumption A1 and A2 can be convert to CFDLc model which is an equivalent dynamical form linearization model.

Theorem 2: Consider nonlinear controller (4), satisfying A1 and A2, if $|\Delta e(k+1)| \neq 0$, then there exists a time-varying parameter $\psi(k) \in R$, called the pseudo-partial- derivative (PPD), such that system (4) can be transformed into the following equivalent dynamic linearization data model, called compact form dynamic linearization based controller (CFDLc) [14]

$$
\Delta u(k)=\psi(k) \Delta e(k+1)
$$

With $|\Delta \psi(k)| \leq \underline{b}_{1}$ for any time $k$.

Proof: Please see reference[14].

Remark 2: Similar with CFDLc, PFDL based controller could be easily derived, and it has been studied in our other work. Like IFT, VRFT in the other DDC methods, the tracking error $e(k+1)$ is not available since the plant model is unknown, instead of a huge amount of measurement $\mathrm{I} / \mathrm{O}$ data of the controlled design becomes an obstacle to the implementation of MFAC method. In this case, a remedy or an intuitive strategy is using a data model, e.g., equivalent CFDL data model, to predict the 
multi-step-ahead system output, consequently the estimated value of the tracking error $e(k+1)$ will be available.

A3b $\psi(k) \leq-\bar{b}_{1}, \forall k \in Z^{+}$, where $\bar{b}_{1}$ is a small positive constant.

Remark 3: The PPD $\psi(k)$ in controller (5) is negative since feedback strategy is used. Moreover, since the controller is artificially designed, the bound $\left[-\underline{b}_{1},-\bar{b}_{1}\right]$ of controller parameters could be known in prior.

Using this assumption and Theorem 2, we have

$$
-\underline{b}_{1} \leq \psi(k) \leq-\bar{b}_{1}<0, \forall k \in Z^{*}
$$

Since controller (5) is the equivalent from of the ideal controller (4) for controlled plant (1), it means that the controller with a qualified parameter estimation algorithm is capable to generate a perfect input signal theoretically to drive the system to get a perfect control performance, that is $e(k+1)=0$. However, it does not mean the actual output tracking error will vanish at one-step ahead consequently due to the uncertainties or estimation error in practice. To make the ideal controller (5) have a causal implementable form in practice and to show the convergence analysis without involving any confusion, we redefine the actual tracking error at instant $k+1$ as $\varepsilon(k+1)=y_{d}(k+1)-y(k+1)$. This leads to the practical controllers based on the equivalent form (5) of the ideal controller (4) as follows

$$
\Delta u(k)=-\psi(k) \varepsilon(k)
$$

If the controlled plant satisfies the following assumptions, then the equivalent compact form dynamic linearization data model of plant (CFDLp) can be obtained.

$\mathrm{A} 4 \mathrm{~b}$ The controller (1) is a smooth nonlinear function, and the partial derivatives of $P(\cdot, \cdot, \cdot)$ with respect to the control input $u(\cdot)$ are continuous.

A5b System (1) is generalized Lipschitz, that is $|\Delta y(k+1)| \leq \bar{b}|\Delta u(k)|$ for any $k$ and $|\Delta u(k)| \neq 0$, where $\Delta y(k+1)=y(k+1)-y(k), \Delta u(k)=u(k)-u(k-1), u(k)=0$ for any $k \leq 0$ and $\bar{b}$ is a positive constant.

Under these assumptions, nonlinear system (1) can be equivalently expressed as following dynamic linearization data model.

$$
y(k+1)=y(k)+\phi(k) \Delta u(k)
$$

Where $\phi(k)$ is the PPD of controlled plant satisfying with $|\phi(k)| \leq \bar{b}$ for any $k$.

The proof of derivation of (8) is similar to the proof of Lemma 1, and is omitted here due to page limited. In next subsection, a type of control method, i.e., CFDLc-MFAPC, is designed for a class of nonlinear system.

\subsection{Design of Predictive Controller Based on Dynamic Linearization of Controller}

When using the practical controller (7), the unknown time-varying PPD parameter must be available before it is utilized in a control loop.

According to (7) and (8), $\mathrm{N}$-step ahead prediction equations are given in follow[10]

$$
\left\{\begin{aligned}
y(k+1) & =y(k)-\phi(k) \varepsilon(k) \psi(k) \\
y(k+2) & =y(k+1)-\phi(k+1) \varepsilon(k+1) \psi(k+1) \\
& =y(k)-\phi(k) \varepsilon(k) \psi(k)-\phi(k+1) \varepsilon(k+1) \psi(k+1) \\
& \vdots \\
y(k+N) & =y(k+N-1)-\phi(k+N-1) \varepsilon(k+N-1) \psi(k+N-1) \\
& =y(k+N-2)-\phi(k+N-2) \varepsilon(k+N-2) \psi(k+N-2) \\
& -\phi(k+N-1) \varepsilon(k+N-1) \psi(k+N-1) \\
& \vdots \\
& =y(k)-\phi(k) \varepsilon(k) \psi(k)-\ldots-\phi(k+N-1) \varepsilon(k+N-1) \psi(k+N-1)
\end{aligned}\right.
$$


Let

$Y_{N}(k)=[y(k+1), \cdots, y(k+N-1)]^{T}$

$\Psi_{N}(k)=\left[\Delta \psi(k), \cdots, \Delta \psi\left(k+N_{u}-1\right), \cdots, \Delta \psi(k+N-1)\right]^{T}$

$E(k)=[1,1, \ldots, 1]^{T}$

$A(k)=\left[\begin{array}{cccccc}\phi(k) \varepsilon(k) & 0 & 0 & \cdots & 0 & 0 \\ \phi(k) \varepsilon(k) & \phi(k+1) \varepsilon(k+1) & 0 & \cdots & 0 & 0 \\ \vdots & \vdots & \ddots & & & \vdots \\ \phi(k) \varepsilon(k) & \cdots & \cdots & \phi\left(k+N_{u}-1\right) \varepsilon\left(k+N_{u}-1\right) & \cdots & \vdots \\ \vdots & \vdots & & \vdots & \cdots & 0 \\ \phi(k) \varepsilon(k) & \phi(k+1) \varepsilon(k+1) & \cdots & \phi\left(k+N_{u}-1\right) \varepsilon\left(k+N_{u}-1\right) & \cdots & \phi(k+N-1) \varepsilon(k+N-1)\end{array}\right]_{N \times N}$

Where $Y_{N}(k+1)$ denotes the N-step ahead prediction vector of the system output, $\Psi_{N}(k)$ is control input increment vector, and $N_{u}$ is the control input horizon.

Then equation (9) can be rewritten in a compact form

$$
Y_{N}(k+1)=E(k) y(k)-A(k) \Psi_{N}(k)
$$

If $\Delta u(k+j-1)=0, j>N_{u}$, then prediction equation (10) becomes

$$
Y_{N}(k+1)=E(k) y(k)-A_{1}(k) \Psi_{N_{u}}(k)
$$

$$
\text { where } A(k)=\left[\begin{array}{cccc}
\phi(k) \varepsilon(k) & 0 & 0 & 0 \\
\phi(k) \varepsilon(k) & \phi(k+1) \varepsilon(k+1) & 0 & 0 \\
\vdots & \vdots & \ddots & \vdots \\
\phi(k) \varepsilon(k) & \phi(k+1) \varepsilon(k+1) & \cdots & \phi\left(k+N_{u}-1\right) \varepsilon\left(k+N_{u}-1\right) \\
\vdots & \vdots & \cdots & \vdots \\
\phi(k) \varepsilon(k) & \phi(k+1) \varepsilon(k+1) & \cdots & \phi\left(k+N_{u}-1\right) \varepsilon\left(k+N_{u}-1\right)
\end{array}\right]_{N \times N_{u}}
$$

In (11), $\phi(k)$ are unknown and have to be replaced by their estimated and predicted values $\hat{\phi}(k)$, in order to implement prediction equation $(11), \phi(k)$ must be estimated first.

\section{Estimation and Prediction of Parameters}

\subsection{Estimation of $\psi(k)$ in CFDLc}

Consider the following cost function with an additional penalty on the abrupt rate of estimated parameter

$$
J(\psi(k))=\sum_{i=1}^{N}\left(y_{d}(k+i)-y(k+i)\right)^{2}+\lambda \sum_{j=0}^{N_{u}-1} \Delta \psi(k)^{2}
$$

Where $\lambda>0$ is weighting factor series. Nothing that $y(k+1)$ is unavailable at instant $k$ since model of controlled plant is unknown.

Let $Y_{N}^{*}(k+1)=\left[y^{*}(k+1), \cdots, y^{*}(k+N)\right]^{T}$, the cost function (12) becomes

$$
J=\left[Y_{N}^{*}(k+1)-Y_{N}(k+1)\right]^{T}\left[Y_{N}^{*}(k+1)-Y_{N}(k+1)\right]+\lambda \Delta \Psi_{N_{u}}^{T}(k) \Delta \Psi_{N_{u}}(k)
$$

Substituting (11) into (13) and using the optimality condition $\frac{\partial J}{\partial \Psi_{N_{u}}(k)}=0$ yield the control law

$$
\Delta \Psi_{N_{u}}(k)=\left[A_{1}^{T}(k) A_{1}(k)+\lambda I\right]^{-1} A_{1}^{T}(k)\left[E(k) y(k)-Y_{d_{N}}^{*}(k+1)\right]
$$

Thus, the control input at current time $k$ is obtained according to the receding horizon principle as follows 


$$
\hat{\psi}(k)=\hat{\psi}(k-1)+g^{T} \Delta \Psi_{N_{u}}(k)
$$

Where $\boldsymbol{g}=[1,0, \cdots, 0]^{T}$.

Thus we need to resort to the CFDLp data model (2), which is an equivalent dynamic data model to the original controlled plant (1).

\subsection{Estimation of $\phi(k)$ in CFDLp}

The value of PPD in CFDLp data model should be obtained by an estimation algorithm for time varying parameter, since it is a time-varying parameter. Here, we use the following modified criterion function to derive the estimation algorithm

$$
J(\phi(k))=(\Delta y(k)-\phi(k) \Delta u(k-1))^{2}+\mu(\phi(k)-\hat{\phi}(k-1))^{2}
$$

Where $\mu>0$ is weighting factor.

Since $A_{1}(k)$ in (15) contains unknown PPD parameters $\phi(k), \phi(k+1), \cdots, \phi\left(k+N_{u}-1\right)$, some time-varying parameter estimation or prediction algorithm should be developed when it is used in applications. Theoretically specking, any estimation algorithm for time- varying parameters can be applied to PPD parameter estimation, but we still use the modified projection algorithm to estimation $\phi(k)$ here in order to facilitate the theoretical analysis for the control system, that is

$$
\hat{\phi}(k)=\hat{\phi}(k-1)+\frac{\Delta u(k-1)}{\mu+\Delta u(k-1)^{2}}[\Delta y(k)-\hat{\phi}(k-1) \Delta u(k-1)],
$$

And $\hat{\phi}(k)$ denotes the estimation of $\phi(k)$.

Since $\phi(k+1), \cdots, \phi\left(k+N_{u}-1\right)$ cannot be calculated from I/O data till sample time $k$ directly, $\phi(k+1), \cdots, \phi\left(k+N_{u}-1\right)$ need to be predicted according to the past estimated sequence $\hat{\phi}(1), \cdots, \hat{\phi}(k)$.For simplicity, the simple two-level prediction method are cited as follows.

Assumed that the estimated values $\hat{\phi}(1), \cdots, \hat{\phi}(k)$ have been calculated by (17) at time $k$. Using these estimated values, an auto-regressive model (AR) for prediction is constructed as follows

$$
\hat{\phi}(k+1)=\theta_{1}(k) \hat{\phi}(k)+\theta_{2}(k) \hat{\phi}(k-1)+\cdots+\theta_{n_{p}}(k) \hat{\phi}\left(k-n_{p}-1\right),
$$

Where $\theta_{i}, i=1, \cdots, n_{p}$ is coefficient, and $n_{p}$ is the fixed model order, which is usually set to be 2 7 as recommened by [10]

Using (18), prediction equation becomes

$$
\hat{\phi}(k+j)=\theta_{1}(k) \hat{\phi}(k+j-1)+\theta_{2}(k) \hat{\phi}(k+j-1)+\cdots+\theta_{n_{p}}(k) \hat{\phi}\left(k+j-n_{p}\right),
$$

Where $j=1, \cdots, N_{u}-1$.

Defining $\theta(k)=\left[\theta_{1}(k), \cdots, \theta_{n_{p}}(k)\right]^{T}$, it is determined by following equation

$$
\theta(k)=\theta(k-1)+\frac{\hat{\varphi}(k-1)}{\delta+\|\hat{\varphi}(k-1)\|^{2}}\left[\hat{\phi}(k)-\hat{\varphi}^{T}(k-1) \theta(k-1)\right],
$$

Where $\hat{\varphi}(k-1)=\left[\hat{\phi}(k-1), \cdots, \hat{\phi}_{n_{p}}\left(k-n_{p}\right)\right]^{T}$, and $\delta \in(0,1]$ is a positive constant.

\subsection{Control Scheme of CFDLc-MFAPC}

Integrating the control law algorithm

$$
\Delta u(k)=-\hat{\psi}(k) \varepsilon(k)
$$

By integrating the control algorithm (21), the parameter estimation algorithm (17), and the prediction algorithm (14) and(15), the CFDL based model-free adaptive predictive control (CFDL-MFAPC) scheme is designed as follows

$$
\begin{gathered}
\Psi_{N_{u}}(k)=\left[\hat{A}_{1}^{T}(k) \hat{A}_{1}(k)+\lambda I\right]^{-1} \hat{A}_{1}^{T}(k)\left[E(k) y(k)-Y_{d_{N}}(k+1)\right] \\
\psi(k)=\psi(k-1)+g^{T} \Delta \Psi_{N_{u}}(k)
\end{gathered}
$$




$$
\begin{gathered}
\hat{\phi}(k)=\hat{\phi}(k-1)+\frac{\Delta u(k-1)}{\mu+\Delta u(k-1)^{2}}[\Delta y(k)-\hat{\phi}(k-1) \Delta u(k-1)], \\
\hat{\phi}(k)=\hat{\phi}(1), \text { if } \hat{\phi}(k)<\hat{\underline{\sigma}} \text { or } \hat{\phi}(k)>\bar{b}
\end{gathered}
$$

Gives the control scheme of CFDLc-MFAPC for the nonlinear system (1). $\lambda_{k}>0, \mu>0, \hat{\underline{\sigma}}$ is a small positive constant, and $\phi(1)$ is the initial value of $\hat{\phi}(k)$.

Remark 4 The reason why we reset the $\hat{\psi}(k)=-\underline{b}_{1}$ as (18) is that the controller gain of $\hat{\psi}(k)$ should not be too small. Otherwise, it cannot be used anymore since it is a controller gain. On the contrary, the controller gain cannot be very large either. Otherwise, it is not implementable or energy saving device. The sign of $\hat{\psi}(k)$ is always negative and $\hat{\phi}(k)$ is positive according to the resetting conditions in algorithm (18)-(20), which are added into this CFDLc-MFAPC scheme to make the scheme have the tracking ability for the time varying parameter.

\section{Simulation Comparison}

In this section, numerical example is given to show the effectiveness and the advantages of CFDLc-MFAPC methods by comparisons with other typical DDC methods, PID, CFDLc-MFAC and MFAC, respectively.

It is worthy pointing out that the following models are only used to generate the $\mathrm{I} / \mathrm{O}$ data, and no any dynamics of the plants is involved in the controller design.

Example Hammerstein system[15]

$$
\begin{aligned}
& x(k)=1.5 u(k)-1.5 u(k)^{2}+0.5 u(k)^{3} \\
& y(k+1)=0.6 y(k)-0.1 y(k-1)+1.2 x(k)-0.1 x(k-1)
\end{aligned}
$$

CFDLc-MFAPC: The initial value and parameter setup of the scheme (26)-(30) is $\hat{\psi}(1)=-0.2$, $\lambda=90$.

CFDLc-MFAC: The initial value and parameter setup is $\hat{\phi}(1)=-0.2, \lambda=1$.

MFAC: The initial value and parameter setup of the scheme is $\hat{\phi}(1)=1, \lambda=1$.

PID: The initial value and parameter setup of the scheme is $k_{p}=0.32, k_{i}=0.18, k_{d}=0.12$.

The comparisons of tracking performance are depicted in Fig.1, and the corresponding control inputs are shown in Fig. 2. The numerical indexes are listed in Table I, including the ITAE

$$
e_{I A E}=\sum_{i}^{4}\left(\sum_{k=1}^{50}\left|e_{i}(k)\right|\right)
$$

And sum of squares of control input rate

$$
\Delta u_{T S S}=\sum_{k=1}^{200} \Delta u^{2}(k)
$$

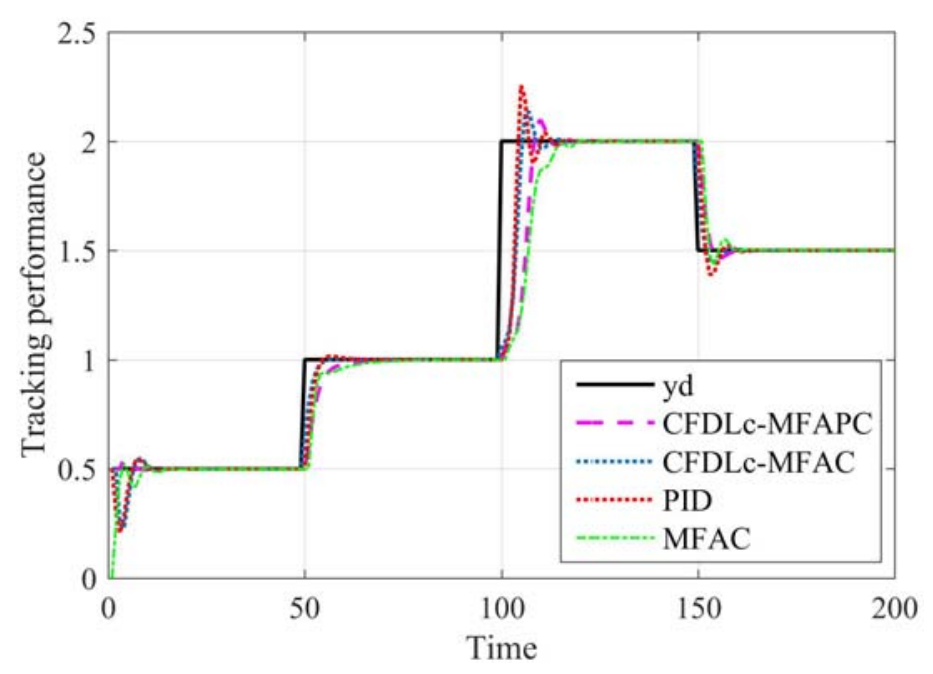

Fig.1. Tracking Performance 


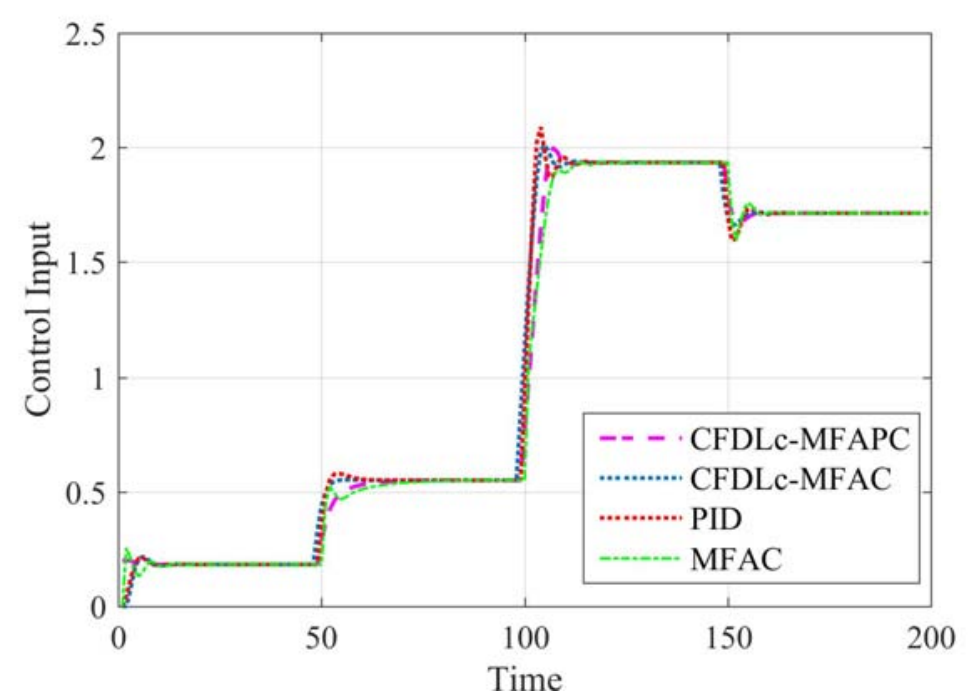

Fig.2. Control Input

Table 1 The indexes of the four methods

\begin{tabular}{ccccc}
\hline & CFDLC-MFAPC & CFDLc-MFAC & MFAC & PID \\
\hline $\boldsymbol{e}_{I A E}$ & 0.6141 & 1.4580 & 0.8718 & 1.607 \\
$\Delta u_{T S S}$ & 0.3470 & 0.7176 & 0.4573 & 0.6099 \\
\hline
\end{tabular}

From Fig.1, it can be seen that the four data driven control methods are effective, and the control performance of CFDLc-MFAPC is superior in terms of the indexes shown in Table I. According to the detail view in Fig. 1, we can also see that the CFDLc-MFAPC has relatively shortest rising time and its overshoot could be neglected, while these specifications in both PID, MFAC and CFDLc-MFAC are not so satisfactory.

\section{Conclusion}

In this work, a novel CFDLc-MFAPC scheme, using dynamic-linearization both on the ideal controller and the controlled plant (CFDLc and CFDLp)of MFAPC, is proposed for a class of nonlinear systems, and parameters of the dynamic linearization data models, both the controller and the controlled plant, are estimated merely utilizing the on-line measured I/O data of the controlled plant. Compared with the existing methods, the control performance is more stable and the control effect is more reliable. The correctness and effectiveness of the proposed scheme is verified by demonstrated by extensive simulation comparisons. Since the entire control system design only depends on the measured I/O data of closed-loop system rather than the first principles model or the identified model, it is a pure data driven or model free control method, and the problems of unmodeled dynamics and conventional robustness in traditional MBC framework are absent.

\section{References}

[1]. Chai T Y, Hou Z S, Lewis F L. Guest Editorial: Special Section on Data-Based Control, Modeling, and Optimization [J]. IEEE TRANSACTIONS ON NEURAL NETWORKS, 2011, 22: 2150-21533.

[2]. Chuanhou G, Ling J, Xueyi L, Jiming C, Youxian S. Data-Driven Modeling Based on Volterra Series for Multidimensional Blast Furnace System [J]. Neural Networks, IEEE Transactions on NEURAL NETWORKS, 2011, 22: 2272-2283.

[3]. Hou Z-S, Wang Z. From model-based control to data-driven control: Survey, classification and perspective [J]. Information Sciences, 2013, 235(Supplement C): 3-35. 
[4]. Hou Z-S, Xu J-X. On data-driven control theory: the state of the art and perspective [J]. ACTA AUTOMATICA SINICA, 2009, 35: 650-667.

[5]. Hou Z. The parameter identification, adaptive control and model free learning adaptive control for nonlinear systems [J]. Shenyang: North-eastern University, 1994.

[6]. Bontempi G, Birattari M, Bersini H. Lazy learning for local modelling and control design [J]. International Journal of Control, 2010, 72(7-8): 643-658.

[7]. Campi M C, Savaresi S M. Direct nonlinear control design: The virtual reference feedback tuning (VRFT) approach [J]. IEEE Transactions on Automatic Control, 2006, 51(1): 14-27.

[8]. Hjalmarsson H. From experiment design to closed-loop control [J]. Automatica, 2005, 41(3): 393-438.

[9]. Karimi A, Mišković L, Bonvin D. Iterative correlation-based controller tuning with application to a magnetic suspension system [J]. Control Engineering Practice, 2003, 11(9): 1069-1078.

[10]. Hou Z, Jin S. Model free adaptive control: theory and applications [M]. CRC press, 2013.

[11]. Hou Z, Liu S, Tian T. Lazy-learning-based data-driven model-free adaptive predictive control for a class of discrete-time nonlinear systems [J]. IEEE transactions on neural networks and learning systems, 2017.

[12]. Hou Z, Liu S, Yin C. Local learning-based model-free adaptive predictive control for adjustment of oxygen concentration in syngas manufacturing industry [J]. IET Control Theory \& Applications, 2016, 10(12): 1384-1394.

[13]. Hou Z, Zhu Y. Controller-dynamic-linearization-based model free adaptive control for discrete-time nonlinear systems [J]. IEEE Transactions on Industrial Informatics, 2013, 9(4): 2301-2309.

[14]. Zhu Y, Hou Z. Controller compact form dynamic linearization based model free adaptive control [C]. Decision and Control (CDC), 2012 IEEE 51st Annual Conference, 2012.

[15]. Yamamoto T, Takao K, Yamada T. Design of a data-driven PID controller [J]. IEEE Transactions on Control Systems Technology, 2009, 17(1): 29-39. 